\title{
5. SCHLUSS \\ DER ABBRUCH DER MODERNEN AUSSENPOLITIK UND BRIANDS EUROPAPLAN
}

Der Erste Weltkrieg bedeutete nicht nur das endgültige Ende des europäischen Konzerts und den Aufstieg der Flügelmächte USA und Sowjetunion, sondern auch eine Modernisierung der Außenpolitik. Neben die nationale Macht- und Interessenpolitik der europäischen Länder, die ihren sichtbarsten Ausdruck im Imperialismus der Vorkriegszeit gefunden hatte, trat ein neues - moderneres außenpolitisches Werte- und Politiksystem. Dieses neue Konzept sah den Zweck der Außenpolitik nicht mehr ausschließlich in der nationalen Interessensicherung, sondern verstärkt in der Wahrung des Friedens und der Sicherheit der Staatengemeinschaft begründet. Die Säulen dieses Friedensmodells waren die Schaffung von kollektiven Sicherheitsstrukturen und die Wiederherstellung des durch den Krieg zerstörten liberalen Weltwirtschaftssystems. Kollektive Sicherheit mit ihren Kernpunkten friedliche Streitschlichtung und Abschreckung sollte internationale Konflikte verhindern und der freie Welthandel die tieferen Ursachen hierfür - Armut und soziale Ungerechtigkeit - beseitigen. Demokratie im Innern der Staaten sollte die Basis dieses modernen, liberalen Friedensmodells bilden. Der wichtigste Propagandist dieses Friedensmodells, der amerikanische Präsident Woodrow Wilson, konnte diese Vorstellungen im Versailler Vertrag allerdings nur unvollständig umsetzen. Das Friedensabkommen blieb in sich widersprüchlich. Modernen Elementen wie vor allem der Völkerbundssatzung und den Bestimmungen über das internationale Arbeitsamt - standen Bestimmungen gegenüber, die traditionellen außenpolitischen Zielsetzungen geschuldet waren. Letztere betrafen vor allem die Abtrennung vieler deutscher Gebiete ohne Volksabstimmung oder die wirtschaftlichen Bestimmungen, die dem Verlierer auferlegt wurden.

Obwohl nach dem Ersten Weltkrieg in Deutschland die Revision des Versailler Vertrags und in Frankreich die nationale Sicherheit vor allem vor Deutschland im Mittelpunkt der jeweiligen außenpolitischen Bemühungen standen, konnten sich auch diese beide Länder nicht völlig der Modernisierung der Außenpolitik entziehen. Sowohl Deutschland als auch Frankreich konnten dabei auf inhaltliche wie organisatorische Entwicklungen aus der Vorkriegszeit zurückgreifen. Die beispielsweise bereits vor dem Krieg angemahnte stärkere Berücksichtigung wirtschaftlicher Belange fand in den administrativen Reformen der auswärtigen Dienste in Deutschland und Frankreich ihren Niederschlag. Insbesondere zwischen den Jahren 1923/24 und 1929 wurden Elemente des liberalen Modells der Friedenssicherung auch in die deutschfranzösischen Beziehungen integriert. 
Bezüglich der Schaffung kollektiver Sicherheitsstrukturen wurden im Untersuchungszeitraum erhebliche Erfolge erzielt: Deutschland trat dem Völkerbund bei, die Locarno-Verträge konstituierten, zumindest für den Bereich der deutschen Westgrenze, ein kollektives Sicherheitssystem, und die Schiedsverträge, die Deutschland - ebenfalls in Locamo - mit seinen östlichen Nachbarn schloß, trugen zur Erhöhung der Sicherheit bei. Ein weiteres wichtiges Element der außenpolitischen Modernisierung wurde mit dem Kriegsächtungspakt erzielt, in dem der Krieg als Mittel der Politik erstmals grundsätzlich verurteilt wurde.

Auch bezüglich der Liberalisierung der Wirtschaftsbeziehungen, dem zweiten wichtigen Aspekt der Modernisierung der Außenpolitik, wurden wichtige Fortschritte erzielt: Durch den deutsch-französischen Handelsvertrag vom 17. August 1927 konnte der bilaterale Handel zwischen Deutschland und Frankreich erleichtert werden. Über die Meistbegünstigungsklausel wirkte diese Liberalisierung auch auf die Handelsbeziehungen Deutschlands und Frankreichs mit anderen Staaten. Auf der Weltwirtschaftskonferenz in Genf im Mai 1927 wurde der freie Handelsverkehr als Ziel der internationalen Wirtschaftspolitik deklariert, mit den Nachfolgekonferenzen erste Schritte auf dem Weg zur Verwirklichung dieses Zieles getan.

Die Modernisierungsanstrengungen machten sich auch in einer zunehmenden Verständigungsbereitschaft zwischen Deutschland und Frankreich bemerkbar, die sich im weitesten Sinne auf die "Zivilgesellschaft ${ }^{1}$ bezog. Auf diese Verständigungsbemühungen kann an dieser Stelle nur exemplarisch ${ }^{2}$

${ }^{1}$ Unter "Zivilgesellschaft« soll dabei »eine Sphäre sozialer Institutionen und Organisationen« verstanden werden, "die nicht direkt der Funktion politischer Selbstverwaltung integriert sind und nicht unmittelbar staatlicher Regulierung unterliegen, doch in verschiedener Weise auf den Staat einwirken: für ihn Grundlagen bereitstellen, Rahmenbedingungen schaffen, seine Leistungen ergänzen, ihn aktiv beeinflussen. In Wirtschaft, Kultur, Bildung, Medien, Verbänden usw. erfullt die Zivilgesellschaft Funktionen, die sich nicht in der Koordinierung von Privatinteressen erschöpfen, sondern die Konstitution eines allgemeinen tragen. Sie bildet kollektive Identität(en), begrüindet Gemeinsinn, stiftet Öffentlichkeit, fördert soziale Sicherheit«, Emil ANGEHRN, Zivilgesellschaft und Staat. Anmerkungen zu einer Diskussion, in: Politisches Denken. Jahrbuch 1992, Stuttgart, Weimar 1993, S. 145-158, hier S. 150. Zur Diskussion um den Begriff der Zivilgesellschaft siehe Hans Manfred BOCK, Das deutschfranzösische Institut in der Geschichte des zivilgesellschaftlichen Austausches zwischen Deutschland und Frankreich, in: DERS. (Hg.), Projekt deutsch-französische Verständigung. Die Rolle der Zivilgesellschaft am Beispiel des Deutsch-Französischen Instituts in Ludwigsburg, Opladen 1998, S. 13-120, hier S. 14-16.

${ }^{2}$ Umfassende bibliographische Angaben zum Thema finden sich in: Hans Manfred BocK, Bibliographischer Versuch zu den zivilgesellschaftlichen Beziehungen zwischen Deutschland und Frankreich im 20. Jahrhundert, in: DERS. (Hg.), Projekt deutsch-französische Verständigung. Die Rolle der Zivilgesellschaft am Beispiel des Deutsch-Französischen Instituts in Ludwigsburg, Opladen 1998, S. 379-477. Weitere bibliographische Hinweise bei Ina BELITZ, Befreundung mit dem Fremden. Die Deutsch-Französische Gesellschaft in den deutsch-französischen Kultur- und Gesellschaftsbeziehungen der Locarno-Ära. Programme 
eingegangen werden, weil sie nicht den eigentlichen Kern des Themas dieser Untersuchung betreffen, der sich ja vor allem auf das Regierungshandeln bezieht. Da aber per definitionem die Zivilgesellschaft mit staatlichem Handeln im Zusammenhang steht, soll dieser Aspekt zumindest kurz angedeutet werden.

Gerade im Umfeld von Locarno kam es zu einer Anzahl von Initiativen, die es sich zur Aufgabe gemacht hatten, das deutsch-französische Verhältnis zu verbessern.

Dazu gehörte unter anderem das Deutsch-Französische Studienkomitee (Comité franco-allemand d'information et de documentation), das durch Einwirkung auf die Presse dazu beitragen wollte, Mißverständnisse und falsche Darstellungen über das jeweils andere Land auszuräumen ${ }^{3}$. Des weiteren wurden bei den Tagungen der Mitglieder verschiedene Aspekte der deutschfranzösischen Beziehungen erörtert ${ }^{4}$. Der Initiator des Studienkomitees, der luxemburgische Industrielle Emile Mayrisch ${ }^{5}$, hatte die Organisation ausdrücklich mit dem Bestreben ins Leben gerufen, den in Locarno eingeleiteten Prozeß zu unterstützen. Das Komitee erhielt dabei sowohl vom Quai d'Orsay als auch vom AA wohlwollende »moralische Unterstützung « ${ }^{6}$.

Insgesamt war eine erstaunliche Vielfalt deutsch-französischer Kontakte festzustellen. Auf wirtschaftlichem Gebiet kam es zu Gesprächen über eine deutsch-französische Kooperation zur Erschließung des französischen Kolonialreiches ${ }^{7}$, die die ausdrückliche Unterstützung Briands hatten, im französischen Kolonialministerium jedoch auf Bedenken stießen ${ }^{8}$. Auch Pläne zu einer

und Protagonisten der transnationalen Verständigung zwischen Pragmatismus und Idealismus, Frankfurt a. M. u.a. 1997 (Diss. Münster 1995, Europäische Hochschulschriften, Reihe 2, 745), S. 535-569. Zusammenfassend siehe Hans Manfred BocK, Kulturelle Eliten in den deutsch-französischen Gesellschaftsbeziehungen der Zwischenkriegszeit, in: Rainer HUDEMANN, Georges-Henri SOUTOU (Hg.), Eliten in Deutschland und Frankreich im 19. und 20. Jahrhundert. Strukturen und Beziehungen, Bd. 1, München 1994, S. 73-91.

3 "L'objet et l'action du Comité français-allemand d'information«, Le Temps (20.6.1926); Aufzeichnung Seydoux (10.11.1925), MAE 1918-1929 Z (Europe) Allemagne, 388. Vgl. BOCK, deutsch-französisches Institut, S. 27-40.

${ }^{4} \mathrm{Vgl}$. Druckschrift "Comité franco-allemand d'information et de documentation « [ca. Anfang 1928] in: MAE PAAP 261, 6.

${ }^{5}$ Vgl. hierzu insbes. Guido MÜLLER, Emile Mayrisch und westdeutsche Industrielle in der westeuropäischen Wirtschaftsverständigung nach dem Ersten Weltkrieg, in: Galerie. Revue culturelle et pédagogique 10/4 (1992), S. 545-559, und DERS., Der luxemburgische Stahlkonzern ARBED nach dem Ersten Weltkrieg. Zum Problem der deutsch-französischen Wirtschaftsverflechtung, in: Revue d'Allemagne et des pays de langue allemande 25/4 (1993), S. 535-543. Dort auch weitere Literaturhinweise.

${ }^{6}$ Runderiaß (ohne Unterschrift) (11.6.1926), PAAA R, 105610. Für Frankreich: Aufzeichnung Seydoux (9.1.1926), MAE 1918-1929 Z (Europe) Allemagne, 388.

${ }^{7}$ Siehe Margerie an Quaj d'Orsay (12.2.1926), MAE 1918-1929 Z (Europe) Allemagne, 390.

${ }^{8}$ Siehe Hoesch an AA (30.11.1926), BArch R 3101, 2640. 
wirtschaftlichen Zusammenarbeit gegenüber der Sowjetunion, die ebenfalls Sympathien auf höchster politischer Ebene fanden, wurden mehrfach erörtert ${ }^{9}$. Weniger ambitiös, aber mit vermutlich höheren Realisierungschancen, waren Pläne zur Gründung einer deutschen Wirtschaftsstelle in Paris und einer deutsch-französischen Handelskammer ${ }^{10}$.

Auch auf kulturellem Gebiet kam es zu Initiativen zur Verbesserung der bilateralen Beziehungen. So sprach sich Painlevé für eine Wiederaufnahme der geistigen Beziehungen zwischen Deutschland und dem Westen aus ${ }^{11}$, und im Sommer 1926 wurde der Boykott, der seit Kriegsende gegenüber der deutschen Wissenschaft bestanden hatte, aufgehoben ${ }^{12}$.

Selbst im militärischen Bereich kam es zu einer gewissen Entspannung: Der französische Militärattaché in Berlin, Oberst René Tournès, traf im November 1929 mit dem Chef der deutschen Heeresleitung, General Wilhelm Heye zusammen ${ }^{13}$. Vermittelt wurde dieses Treffen durch den deutschen General Georg von der Lippe, der wiederum mit Arnold Rechberg bekannt war. Rechberg war als Propagandist eines deutsch-französischen Bündnisses hervorgetreten, das dazu beitragen sollte, Europa vor der Gefahr des Bolschewismus zu schützen. Allerdings sollte dieses Bündnis erst dann zustande kommen, wenn einige wesentliche deutsche Revisionsziele erfüllt worden wären. Nichtsdestotrotz wertete Tournès die Gespräche positiv, da sie beitragen könnten, unter der deutschen Rechten - Lippe war Mitglied im "Stahlhelm« und in der DNVP - die Feindschaft gegenüber Frankreich abzubauen ${ }^{14}$.

Auch auf parteipolitischer Ebene gab es Fortschritte. Wenig erstaunlich war dies bei den Sozialisten, die traditionell internationalistisch eingestellt waren. Hier kam es zu verschiedenen Treffen zwischen Politikern mehrerer europäischer Länder, unter ihnen auch Franzosen und Deutsche ${ }^{15}$. Der ehemalige Minister Le Troquer schlug im Sommer 1928 die Gründung einer deutschfranzösischen Parlamentariergruppe vor, der allerdings in Frankreich vor al-

${ }^{9}$ Siehe Aufzeichnung ohne Unterschrift (2.3.1926), MAE 1918-1929 Z (Europe) Allemagne, 391; Margerie an Quai d'Orsay (7.12.1928), MAE 1918-1929 Z (Europe) Allemagne, 392.

${ }^{10}$ Siehe Aufzeichnung ohne Unterschrift (5.11.1928), BArch R 3101, 2644/1.

11 Siehe Hoesch an AA (3.6.1926), PAAA R, 70527.

${ }^{12}$ Siehe Brigitte SCHROEDER-GUDEHUS, Die Jahre der Entspannung. Deutsch-französische Wissenschaftsbeziehungen am Ende der Weimarer Republik, in: Yves COHEN, Klaus MANFRASS ( $\mathrm{Hg}$.), Frankreich und Deutschland. Forschung, Technologie und industrielle Entwicklung im 19. und 20. Jahrhundert. Internationales Kolloquium, veranstaltet vom Deutschen Historischen Institut Paris in Verbindung mit dem Deutschen Museum München und der Cité des Sciences et de l'Industrie Paris, München, 12.-15. Oktober 1987, München 1990, S. 105-115, hier S. 106.

${ }^{13}$ Siehe Tournès an $2^{\mathrm{e}}$ Bureau (30.11.1928), MAE 1918-1929 Z (Europe) Allemagne, 392. Siehe auch zum folgenden.

${ }^{14}$ Siehe Tournès an $2^{e}$ Bureau (17.4.1929), MAE 1918-1929 Z (Europe) Allemagne, 392.

${ }^{15}$ Siehe Hoesch an AA (29.12.1927), PAAA R, 28243. 
lem linke und Mittelparteien angehörten ${ }^{16}$. Einige Reichstagsabgeordnete aus SPD, DVP, DDP, Wirtschaftspartei und Zentrum fanden sich nach anfänglichem Zögern schließlich bereit, eine deutsche Partnerorganisation zu grün$\operatorname{den}^{17}$. Eine erste gemeinsame Sitzung fand am 31. Mai 1929 in Paris statt ${ }^{18}$.

Zwischen französischen und deutschen Katholiken kam es auf Anregung des Studienkomitees ebenfalls zu Treffen, mit denen die Hoffnung verbunden wurde, "daß die Anknüpfung vertrauensvollerer Beziehungen zwischen dem deutschen und französischen Katholizismus ein wertvolles Agens in den politischen Beziehungen beider Völker sein kann und wird « ${ }^{19}$. Interessant waren diese Gespräche wegen der »enge[n] Verbindung in Frankreich zwischen Katholizismus und Nationalismus $"{ }^{20}$ und weil $»$ Katholizismus und Reaktion in Frankreich traditionell weitgehend identisch sind ${ }^{21}$. Da das deutsche Zentrum seinen Schwerpunkt im zum Teil noch immer französisch-besetzten Rheinland hatte, konnte man in diesen Treffen tatsächlich einen wichtigen Schritt Richtung deutsch-französischer Annäherung sehen. Nach einer neuerlichen $\mathrm{Zu}$ sammenkunft zwischen deutschen und französischen Katholiken im Dezember 1929 gewann Hoesch den Eindruck, daß dieses Treffen »ein voller Erfolg gewesen ist. Seine Folgen werden sich immer deutlicher auswirken, und das ist um so höher zu bewerten, als er sich auf verhältnismässig [sic] rechtsstehende Kreise erstreckt, die der Annäherungspolitik bisher ziemlich ablehnend gegenüber standen (22 $^{22}$.

Selbst die extreme Rechte entwickelte Ideen zu einer Annährung. Der Reichstagsabgeordnete Moritz Klönne (DNVP) schlug eine deutschfranzösisch-britische Zusammenarbeit vor, wenn zuvor weitreichende deutsche Revisionsforderungen erfüllt würden ${ }^{23}$. Kapitän Ehrhardt, als Freikorpsführer zu einer gewissen Berühmtheit gelangt, sprach sich ebenfalls für eine Annäherung an Frankreich aus ${ }^{24}$. Wie bei Rechberg waren diese Überlegungen antibolschewistisch motiviert ${ }^{25}$. In Frankreich fand dieser politische Ansatz unter anderem Zustimmung bei Robert Fabre-Luce und Paul Reynaud ${ }^{26}$.

Die Fortschritte in den Bereichen der kollektiven Sicherheit, der wirtschaftlichen Kooperation und auch der gesellschaftlichen Annäherung sind um so

\footnotetext{
${ }^{16}$ Siehe Aufzeichnung Corbin (19.6.1928), MAE 1918-1929 Z (Europe) Allemagne, 391.

${ }^{17}$ Siehe Wirth an Le Trocquer (23.3.1929), PAAA R, 70534.

${ }^{18}$ Siehe Aufzeichnung Deslaurens [31.5.1929], PAAA R, 70534.

${ }^{19}$ Aufzeichnung Papen (o.D.), PAAA R, 70533.

${ }^{20}$ Aufzeichnung ohne Unterschrift (14.9.1928), PAAA R, 70533.

${ }^{21}$ Ibid.

${ }^{22}$ Hoesch an AA (14.1.1930), PAAA R, 70535.

${ }^{23}$ Siehe Guerlet an Quai d'Orsay (23.9.1929), MAE 1918-1929 Z (Europe) Allemagne, 392.

${ }^{24}$ Siehe Hoesch an AA (24.12.1929), PAAA R, 70535.

${ }^{25}$ Siehe ibid.; Guerlet an Quai d'Orsay (23.9.1929), MAE 1918-1929 Z (Europe) Allemagne, 392.

${ }^{26}$ Siehe Aufzeichnung Bassenheim (12.11.1926), PAAA R, 70528.
} 
erstaunlicher vor dem Hintergrund der schwierigen gesellschaftlichen, wirtschaftlichen und internationalen Rahmenbedingungen. Auch die Kürze der Zeit, in der diese Fortschritte erreicht wurden, belegt, daß die Modernisierung der Außenpolitik eine beträchtliche Wirkung entfaltete.

Trotz all dieser Fortschritte blieb die Modernisierung der Außenpolitik während des gesamten Untersuchungszeitraumes jedoch stark beschränkt und der eingeleitete Modernisienungsproze $B$ war nicht unumkehrbar. Wie bereits dargelegt wurde, blieben die Schlichtungs- und Sanktionsmechanismen des Völkerbunds fakultativ und wurden nicht konkretisiert. Versuche dazu, wie beispielsweise das Genfer Protokoll, scheiterten. Auch Locarno beinhaltete keine konkreten Pläne für den Emstfall und war außerdem regional begrenzt. Der potentiell größere Konfliktherd - die deutsche Ostgrenze - blieb von internationalen Sicherheitsgarantien weitgehend ausgenommen. Ebenso führte der Kriegsächtungspakt zu keiner materiellen Verbesserung der Sicherheitslage. Trotz aller Fortschritte seit Kriegsende gab es auch am Ende der 1920er Jahre ein europäisches Sicherheitsproblem.

Auch im außenwirtschaftlichem Bereich blieben die Erfolge der Modernisierung bescheiden: Selbst nach dem deutsch-französischen Handelsvertrag blieb das Zollniveau zwischen den Staaten hoch, auf internationaler Ebene kam es sogar bereits ab 1928 wieder zu steigenden Tarifen. Der wirtschaftsliberale Impetus, der von der Genfer Weltwirtschaftskonferenz ausging, verpuffte in den Nachfolgekonferenzen weitgehend, wie das Scheitern der Konvention über die Beseitigung von Handelshemmnissen zeigte. Die internationale Kartellierung der Nachkriegszeit führte zur Ausklammerung wichtiger Produkte aus der eigentlichen Handels- und Zollpolitik und relativierte damit die ohnehin begrenzten Ansätze zur Liberalisierung der Weltwirtschaft weiter. Viele deutsch-französische Projekte, wie beispielsweise die koloniale Zusammenarbeit oder eine wirtschaftliche Kooperation gegenüber Rußland, kamen über das Stadium von Vorüberlegungen kaum hinaus.

Auch die oben genannten zivilgesellschaftlichen Verständigungsbemühungen blieben insgesamt doch recht bescheiden. Es fällt auf, daß es sich bei den Aktiven der verschiedenen Organisationen vielfach um die gleichen Personen handelte ${ }^{27}$. Diese Gruppierungen waren außerdem keine Massenorganisationen, sondern kleine, begrenzte Zirkel $^{28}$. In der Öffentlichkeit wurden diese Verständigungsbemühungen darüber hinaus kaum wahrgenommen. Die Besu-

${ }^{27}$ Siehe "Organisations françaises pour l'entente européenne«, Europäische WirtschaftsUnion (1.4.1928).

${ }^{28}$ Siehe Laurence BADEL, Les promoteurs français d'une union économique et douanière de l'Europe dans l'entre-deux-guerres, in: Antoine FLEURY, Lubor JILEK ( $\mathrm{Hg}$.), Le Plan Briand d'Union fédérale européenne. Perspectives nationales et transnationales, avec documents. Actes du colloque tenu à Genève du 19 au 21 septembre 1991, Bern u.a. 1998, S. 17-29, hier S. 29. 
che Thomas Manns und Alfred Kerrs in Paris verliefen weitgehend unbeach$\operatorname{tet}^{29}$. Herriot, der sich öffentlich zur deutsch-französischen Aussöhnung bekannte, sah sich deswegen in Paris immer noch den mübelsten Anpöbelungen ${ }^{30}$ ausgesetzt. Viele Verständigungsbemühungen, wie beispielsweise die des deutsch-französischen Studienkomitees, litten an einer schlechten finanziellen Ausstattung ${ }^{31}$, und innerhalb dieser Organisation kam es zu Spannungen: Viele Franzosen befürchteten, daß deutsche Industrielle das Studienkomitee dazu benutzen wollten, eine »hégémonie économique « ${ }^{32}$ zu errichten.

Insgesamt gelangt man zu dem Eindruck, daß bei diesen verschiedenen Initiativen vielfach nur die jeweiligen nationalen Standpunkte dargelegt wurden, ohne da $\mathrm{B}$ es wirklich zur Erörterung von praktikablen Lösungsmöglichkeiten $\mathrm{kam}^{33}$. Allerdings gilt auch für diesen Bereich der deutsch-französischen Beziehungen, daß der offene Meinungsaustausch und die Gesprächsbereitschaft nur wenige Jahre nach dem Krieg bereits einen erheblichen Fortschritt an sich bedeuteten.

Woran lag es, daß die Modernisierung der Außenpolitik in den deutschfranzösischen Beziehungen relativ beschränkt blieb? Eine wichtige Ursache war, daß sie sowohl in Deutschland als auch in Frankreich stets nur eine auBenpolitische Option war. Frankreich verfolgte je nach politischer Konjunktur noch (mindestens) zwei andere außenpolitische Strategien: Die Bündnispolitik und - vielleicht prononcierter als gemeinhin angenommen wird - eine Politik der eigenen Stärke. Auch nach Locarno waren diese beiden Konzepte - wie das Zusammengehen zwischen England und Frankreich in Abrüstungsfragen und die ursprünglichen französischen Pläne zum Briand-Kellogg-Pakt zeigten - bedeutsam. Die Projekte zur Befestigung der französischen Ostgrenzen Stichwort Maginot-Linie - verdeutlichten, daß Frankreich seine Sicherheit

\footnotetext{
${ }^{29}$ Siehe Hoesch an AA (20.8.1927), PAAA R, 28242.

${ }^{30}$ Ibid.

${ }^{31}$ Siehe Seydoux an Margerie (23.5.1927), MAE PAAP 261, 42. Poincaré selbst versuchte, die französischen Großbanken zu stärkerem finanziellen Engagement zu bewegen. Diese hielten sich jedoch zurück: Sie forderten, daß erst die Gesetze abgeschafft werden müßten, die die Betätigung französischer Banken im Ausland verboten, und die Bestimmungen des Versailler Vertrags zur Beschlagnahme deutschen Eigentums in Frankreich aufgehoben würden. Erst dann könnten sich die Banken auch tatkräftig am wirtschaftlichen Austausch und der Verständigung beteiligen, siehe Moret an Celier (20.5.1927), Ex-BNP, 41688-4; Aufzeichnung ohne Unterschrift [Moret?] (8.6.1927), Ex-BNP, 41688-4.

${ }^{32}$ Aufzeichnung Seydoux (15.6.1926), MAE 1918-1929 Z (Europe) Allemagne, 388.

${ }^{33}$ Siehe beispielsweise das Interview mit Bruhn, Mitglied des deutsch-französischen Studienkomitees und Generaldirektor bei Krupp, in dem dieser die deutschen Revisionsforderungen (Rückgabe des Korridors, Truppenabzug usw.) wiederholte: „Um die deutschfranzösische Verständigung«, Kölnische Volkszeitung (6.6.1926). In dem Gespräch zwischen Heye und Tournès legte der deutsche General ebenfalls die deutschen Revisionswünsche dar, auf die Tournès jedoch kaum einging, siehe Tournès an $2^{\mathrm{C}}$ Bureau (30.11.1928), MAE 1918-1929 Z (Europe) Allemagne, 392.
} 
weiterhin stärker durch die eigene Verteidigungsbereitschaft als durch den deutschen Verständigungswillen gewährleistet sah.

Für die deutsche Revisionspolitik wiederum spielte auch nach Locarno die wrussische Option« eine Rolle, wie der Berliner Vertrag und die Bemühungen zeigten, die sowjetischen Bedenken hinsichtlich eines deutschen Völkerbundsbeitritts zu zerstreuen. Mit anderen Worten: Eine moderne Außenpolitik - im Sinne der Herstellung von kollektiver Sicherheit, Stabilität und Wohlstand - war bis 1929 nicht so sehr das Ziel der deutschen und der französischen Außenpolitik, sondern nur eine von mehreren Methoden zur Erreichung anderer außenpolitischer Ziele, nämlich zur Erlangung von sécurité einerseits und zur Durchsetzung von Revisionsforderungen andererseits. Allerdings darf der Einsatz moderner außenpolitischer Methoden nicht so verstanden werden, $\mathrm{da} ß$ es sich dabei lediglich um ein rein taktisches Element zur Umsetzung sicherheits- und revisionspolitischer Zielsetzungen handelte ${ }^{34}$. Weder Deutsche noch Franzosen machten einen Hehl aus ihren außenpolitischen Zielen, so daß nicht davon ausgegangen werden kann, daß die Verständigungspolitik genutzt wurde, um ihre eigentlichen politischen Absichten zu verschleiern. Beide Regierungen versuchten außerdem, ihre außenpolitischen Ziele mit den modernen Methoden in Einklang zu bringen. Sowohl die deutsche Revisions- als auch die französische Sicherheitspolitik veränderten sich unter dem Einfluß des liberalen Modells nicht unerheblich: Deutschland entwickelte ein Revisionsmodell, das verstärkt auf friedliche Mittel setzte ${ }^{35}$, und Frankreich gestaltete seine Sicherheitspolitik so, daß sie mehr auf deutsche Befindlichkeiten Rücksicht nahm, indem es stärker auf die Finanzdiplomatie setzte und auf das Rheinland als strategisches Glacis verzichtete. Soutou kommt zu dem Schluß, daß seit Locarno »aucun dirigeant français n'échappe complètement au dogme de la sécurité collective ${ }^{36}$. Dennoch konnten die latenten Widersprüche zwischen französischem Sicherheitsstreben, deutschem Revisionsverlangen und liberalem Modell in den 1920er Jahren nicht völlig ausgeglichen werden, worin letztlich auch eine der Ursachen für das Scheitern der modernen Außenpolitik lag.

Dabei darf jedoch nicht vergessen werden, daß die Mittel und die Möglichkeiten der modernen Außenpolitik im Untersuchungszeitraum noch neu und unerprobt waren. Es bedurfte eines langen Lern- und Anpassungsprozesses, um Widersprüche zwischen Methoden und Zielen aufzuspüren und die richti-

\footnotetext{
${ }^{34}$ Diese These vertritt beispielsweise Hagspiel, siehe Hermann HaGSPIEL, Die Auffassung und die Benützung von Konzepten der rneuen Diplomatie ( in der deutsch-französischen Verständigungspolitik (1924-1928), in: Jacques BARIÉTY, Antoine FLEURY (Hg.), Mouvements et initiatives de paix dans la politique internationale: 1867-1928. Actes du colloque tenu à Stuttgart 29-30 août 1985, Bern 1987, S. 335-353, hier S. 351.

${ }^{35}$ Siehe Kap. 4.1.4.

${ }^{36}$ SOUTOU, Sécurité collective, S. 131.
} 
gen Konsequenzen daraus zu ziehen, zumal die Komplexität der internationalen Beziehungen durch die enge Verknüpfung wirtschaftlicher, politischer und sozialer Fragen extrem zugenommen hatte.

Das Scheitern der modernen Außenpolitik Ende der 1920er Jahre war deshalb keine Zwangsläufigkeit. Die verständigungsorientierte Politik hatte im Gegenteil dazu beigetragen, viele Probleme, die sich unmittelbar aus dem Krieg und dem Versailler Vertrag ergeben hatten, zumindest vorläufig zu lösen und zu einem modus vivendi zu gelangen. Das Reparations- und Schuldenproblem wurde durch den Dawes- bzw. Young-Plan entschärft. Durch die deutsche Entwaffnung und die Demilitarisierung des Rheinlandes sowie durch Locarno kam es zu Fortschritten im Bereich der Sicherheit. Die Lösung dieser Fragen wurde innerhalb des Rahmens der Bestimmungen des Versailler Vertrags erzielt und stellte diesen nicht grundsätzlich in Frage.

In eine kritische Phase trat die Modernisierung jedoch, als die Folgeprobleme des Versailler Vertrags gelöst worden waren und die grundsätzliche Entscheidung und Neubewertung darüber näherrückte, ob und wie Sicherheitsbzw. Revisionspolitik weiter betrieben werden und in welchem Verhältnis diese beiden Ziele zu einer modernen Außenpolitik stehen sollten.

Nach der Haager Konferenz - und erst dann - konnte darüber verhandelt werden, wie die europäische Nachkriegsordnung (mit dem deutschfranzösischen Verhältnis als zentralem Problem) grundsätzlich ausgestaltet werden sollte. Erst zu diesem Zeitpunkt, Ende 1929/Anfang 1930, stand die Frage an, welche grundsätzlichen Ziele die französische und deutsche Außenpolitik verfolgen sollte. Das zentrale Problem dabei war: Würden Deutschland der Revision und Frankreich der Sicherheit als Oberziel ihrer Außenpolitik treu bleiben, oder würden diese beiden Ziele einer gesamteuropäische Friedensordnung auf Grundlage des liberalen Modells untergeordnet? Anders als Knipping ${ }^{37}$, Niedhart ${ }^{38}$ und andere ${ }^{39}$ sehe ich das Jahr 1927/28 nicht als Wendejahr der internationalen Beziehungen, von dem an die Verständigungspolitik langsam abzubröckeln begann, sondern vielmehr als eine Plateau-Phase, in der es um die grundsätzliche Neuorientierung der Außenpolitik ging. Zur Jahreswende 1929/1930 waren das europäische Staatensystem und die deutschfranzösischen Beziehungen also in einer äußerst labilen Entscheidungssituation, weil es zu einer prinzipiellen Festlegung des Verhältnisses von Zielen und Methoden der zukünftigen deutschen und französischen Außenpolitik kommen mußte.

In dieser Situation kam dem Europa-Plan Briands eine besondere Bedeutung zu, weil darin ein mögliches Zukunftskonzept für das internationale Staatensystem (zumindest für dessen europäischen Teil) entwickelt wurde. Die deutsche

\footnotetext{
${ }^{37}$ Siehe KNIPPING, Locarno-Ära, S. 32.

${ }^{38} \mathrm{Vgl}$. NIEDHART, Intemationale Beziehungen, S. 80-87.

${ }^{39}$ Siehe JACOBSON, Locarno Diplomacy, S. 101; BUCHHEIT, Briand-Kellogg-Pakt, S. 138.
} 
Stellungnahme zum französischen Europa-Memorandum gibt deshalb - zumindest begrenzt - Auskunft darüber, welche Ziele Deutschland in Zukunft verfolgen würde.

Die Beurteilung der Intentionen, die Briand und die französische Außenpolitik zu ihrer Europainitiative bewegten, wird vor allem dadurch erschwert, da $\beta$ es nur wenige Anhaltspunkte gibt, die »die Hintergründe dieser französischen Initiative $[\ldots]$ erhellen ${ }^{40}{ }^{4}$. Nichtsdestotrotz läßt sich sagen, daß sich der briandsche Europaplan aus drei Quellen speiste: Erstens, den verschiedenen Zollunionsplänen, zweitens, der französischen Sicherheitspolitik und, drittens, im weitesten Sinne idealistischen Einstellungen ${ }^{41}$. Trotz der oftmals problematischen politischen Beziehungen zwischen Deutschland und Frankreich zirkulierten verschiedene Pläne für eine Zollunion zwischen beiden Staaten. Auf französischer Seite hatten vor allem Bosc und Molinari eine Zollunion für möglich gehalten, da sich die beiden Volkswirtschaften auf ähnlichem Niveau befänden und sich gegenseitig ergänzten ${ }^{42}$. Hugo Stinnes sprach sich unmittelbar nach dem Ersten Weltkrieg für eine deutsch-französische Zollunion aus, von der er Vorteile für die deutsche Industrie erwartete. Im Zusammenhang mit dem Auslaufen der Wirtschaftsbestimmungen des Versailler Vertrags zum 10. Januar 1925 begann sich das Reichswirtschaftsministerium ebenfalls mit einer deutsch-französischen Zollunion zu beschäftigen. Aus wirtschaftlichen Gründen hielt man diese für durchaus wünschenswert und technisch machbar. Zwei Gründe sprachen aber nach Ansicht des RWiM dagegen: Eine deutschfranzösische Zollunion würde wahrscheinlich dazu führen, daß die Exporte Englands und der USA nach Frankreich durch deutsche Produkte ersetzt würden, weshalb »die übrige Welt ein solches Gebilde bekämpfen ${ }^{43}$ würde. Dadurch würde die europäische Wirtschaft nach den Kriegsfolgen und dem Ruhrkampf erneut in "Unruhen ${ }^{44}$ versetzt, die insgesamt als schädlich beurteilt wurden. Eine weitere Schwierigkeit sah das RWiM darin, daß eine Zollunion die gemeinsame Planung der Zollpolitik und darüber hinaus auch anderer Bereiche der Wirtschaftspolitik notwendig machen würde. Frankreich allein darüber entscheiden zu lassen, war aus deutscher Sicht eine »unmögliche Vorstellung « und eine "kulturwidrige Konstruktion ${ }^{45}$. Ein gemeinsames Zollparlament zur Regelung dieser Fragen würde dagegen wohl in Frankreich auf Ablehnung stoßen: "Rein politisch betrachtet ist Frankreich für die Vor-

\footnotetext{
${ }^{40}$ KNIPPING, Locarno-Ära, S. 85.

${ }^{41}$ Siehe ibid. Ähnlich argumentieren WRIGHT, Stresemann, S. 483; SCHULZ, Wirtschaftsordnung, S. 180; KRÜGER, Schwierigkeit, S. 123f.

${ }^{42}$ Zum folgenden siehe undatierte Aufzeichnung ohne Unterschrift [1924], BArch R 3101, 20458.

${ }^{43}$ Ibid.

${ }^{44}$ Ibid.

${ }^{45} \mathrm{Ibid}$.
} 
stellung eines gemeinsamen [Zoll-, R.B.] Parlamentes heute nicht reif ${ }^{46}$. Für den Moment sah man also in Deutschland eine Zollunion zwar prinzipiell für wünschenswert aber nicht realisierbar an. Stresemann selbst sprach sich allerdings gegenüber Margerie dafür aus, ein solches Projekt etwa zwischen 1930 und $1935 \mathrm{zu}$ realisieren ${ }^{47}$.

Eine deutsch-französische bzw. eine europäische Zollunion hatte aber nicht nur Anhänger in Deutschland, sondern auch in Frankreich. Hier hatte die Union douanière européenne (UDE) eine gewisse Bedeutung. Sie war am 12. März 1925 nach einem "Aufruf an die Europäer« entstanden, den neben dem Franzosen Charles Gide unter anderem auch der Deutsche Edgar SternRubarth, der wiederum ein Vertrauter Stresemanns war $^{48}$, sowie der Ungar Elemér Hantos unterzeichnet hatten ${ }^{49}$. Norman Angell, der bereits vor dem Ersten Weltkrieg die Idee popularisiert hatte, daß weltwirtschaftliche Verflechtung Konflikte verhindern würde, weil diese ökonomisch sinnlos seien, war ebenfalls Mitglied ${ }^{50}$. Die UDE erlangte vor allem deshalb einen gewissen Einfluß, weil sie über gute Kontakte zum Quai d'Orsay verfügte ${ }^{51}$ und weil sie viele bedeutende Persönlichkeiten zu ihren Mitgliedern zählen konnte. Briand selbst war Ehrenpräsident, der ehemalige Minister Yves Le Trocquer Vorsitzender $^{52}$. Seydoux saß im Verwaltungsrat, und zu den Mitgliedern zählten zahlreiche ehemalige Minister, Parlamentarier und Professoren. Der Nachfolger Serruys im französischen Handelsministerium, Paul Elbel, war ebenfalls Mitglied. War die Zielsetzung der Union douanière anfangs auf den globalen Abbau von Handelshemmnissen ausgerichtet, verlagerte sich ihr Arbeitsschwerpunkt später zunehmend auf die Forderung nach einer regionalen wirtschaftlichen Kooperation in Europa ${ }^{53}$. Einen erneuten deutschen Vorstoß in dieser Frage unternahm Wilhelm Eggert, der als Vertreter des ADGB an der Genfer Weltwirtschaftskonferenz teilnahm. Dort schlug er - im Beisein Trendelenburgs - vor, zunächst zu einer Vereinheitlichung der Zollsätze und der Zollnomenklatur zu kommen, um anschließend über den schrittweisen Abbau der Zölle schließlich eine europäische Zollunion zu schaffen ${ }^{54}$. Diese Zollunion sollte wiederum Vorstufe für die Verwirklichung des weltweiten Freihandels sein. In der Tat: „Dies war nichts geringeres als die Vorwegnahme des

${ }^{46}$ Tbid

${ }^{47}$ Siehe Margerie an Quai d'Orsay (29.8.1925), MAE 1918-1929 Z (Europe) Allemagne, 524.

${ }^{48}$ Siehe SCHONEBERGER, Diplomatie, S. 55, 69, 99.

${ }^{49}$ Siehe BADEL, Promoteurs, S. 18.

${ }^{50}$ Siehe Aufzeichnung Corbin [?] (19.11.1928), MAE 1918-1940 Y (Internationale), 635.

${ }^{51}$ Siehe BADEL, Promoteurs, S. 18, 23.

52 Siehe „Organisations françaises pour l'entente européenne«, Europäische WirtschaftsUnion (1.4.1928); siehe auch zum folgenden.

${ }^{53}$ Siehe BADEL, Promoteurs, S. 18.

${ }^{54}$ Siehe SCHULZ, Wirtschaftsordnung, S. 96. 
Briand-Planes auf der Ebene der europäischen Handelsordnung “ ${ }^{55}$, wurde jedoch von Frankreich und England abgelehnt. Trotz dieser Ablehnung blieb eine europäische Zollunion weiterhin in der Diskussion. Im Januar 1929 äuBerte sich Serruys gegenüber Hoesch besorgt, daß Deutschland von der europäischen Wirtschaftskooperation zugunsten einer noch engeren Zusammenarbeit mit den USA abweichen könnte ${ }^{56}$. Eine Befürchtung, die Ritter für unbegründet hielt:

Es ist nach wie vor eines der Hauptziele unserer Wirtschaftspolitik, die wirtschaftliche Verständigung innerhalb Europas zu fördern. Zur Erreichung dieses Zieles ist möglichst weitgehender Wirtschaftsausgleich zwischen Deutschland und Frankreich eine wichtige Voraussetzung. [...] Leider sind wir indessen bei der Verwirklichung dieser beiden Absichten durch zwei ungelöste Fragen erheblich behindert: Reparationen und Răumung ${ }^{57}$.

Aus französischer Sicht - dies ist für den Zeitpunkt der Veröffentlichung der briandschen Europapläne von Bedeutung - war nach der vermeintlich erfolgreichen Lösung dieser beiden »ungelösten Fragen« auf der Haager Konferenz der Moment gekommen, die politische und wirtschaftliche Ordnung Europas weiter auszugestalten.

Angestoßen durch die zunehmende Aktivität Briands auf dem Gebiet der europäischen Kooperation wurden auch vom Völkerbund Pläne für eine stärkere wirtschaftliche Zusammenarbeit der europäischen Länder entwickelt: Sir Arthur Salter, der Direktor der Section économique im Völkerbundssekretariat, und sein Stellvertreter, Pietro Stoppani, sprachen sich während der Bundesversammlung, auf der Briand seinen Europa-Plan vortragen wollte, für eine wirtschaftliche Stärkung Europas aus. Stoppani ${ }^{58}$, der in der wirtschaftlichen Zersplitterung Europas das Hauptübel für die schlechte Wirtschaftslage sah, schlug die Schaffung eines gemeinsamen europäischen Marktes nach Vorbild der USA vor. Allerdings sollte dies erst das Endziel einer langfristigen Entwicklung sein. Zunächst sollten die Zolltarife eingefroren und später schrittweise gesenkt werden. Stoppani sprach sich außerdem dafür aus, zunächst die Industrieländer zusammenzufassen, und die stärker agrarisch geprägten Staaten Ost- und Südosteuropas erst später, nach Lösung von deren Wirtschaftsproblemen, aufzunehmen. Die Pläne Salters bewegten sich in eine ähnliche Richtung. Auch er forderte einen zweijährigen Zollfrieden, dem Zollsenkungen folgen sollten ${ }^{59}$. Allerdings schien das Endziel Salters weniger ein gemeinsamer europäischer Markt zu sein, sondern vielmehr ein europäisches

\footnotetext{
${ }^{55}$ Ibid. S. 97.

${ }^{56}$ Siehe Hoesch an AA (5.1.1929), ADAP B XI, Nr. 8.

${ }^{57}$ Ritter an Botschaft Paris (31.1.1929), ADAP B XI, Nr. 43.

${ }^{58} \mathrm{Zu}$ den Vorschlägen Stoppanis siehe BUSSIĖRE, Organisation économique, S. 308f; DERS., Aspects économiques, S. 86.

${ }^{s 9}$ Siehe Aufzeichnung Imhoff (6.9.1929), ADAP B XIII, Nr. 5.
} 
Präferenzsystem mit Ausrichtung vor allem gegen die »amerikanische Gefahr ${ }^{60}{ }^{6}$ und Vorkehrungen dagegen, daß die USA einseitig von der Meistbegünstigungsklausel profitierten.

Im Europa der 1920er Jahre war die Idee einer Zollunion also durchaus verbreitet und dürfte - die Verbindung zwischen Briand und der UDE belegt dies - sicherlich eine wichtige Quelle für das französische Europamemorandum gewesen sein.

Eine weitere Motivation ergab sich sicherlich aus dem nach wie vor ungestillten französischen Sicherheitsbedürfnis. Locarno und der Briand-KelloggPakt stellten, wie bereits dargelegt wurde, noch keineswegs die umfassenden Sicherheitsgarantien dar, die Frankreich anstrebte ${ }^{61}$. Das Abkühlen des französisch-britischen Verhältnisses mit dem Regierungsantritt MacDonalds im Juni 1929 dürfte die französische Regierung dazu veranlaßt haben, mit dem Europaplan auch eine neue Initiative in der Sicherheitspolitik zu starten ${ }^{62}$.

Naturgemäß am schwierigsten dürfte die Beantwortung der Frage nach den ideellen Grundlagen des briandschen Europamemorandums sein. Dennoch gibt es Anhaltspunkte dafür, daß der Europaplan nicht nur Resultat eines wirtschaftlichen und sicherheitspolitischen Kalküls war, sondern auch idealistische Momente enthielt. Ein Element für den Friedenswillen Briands waren sicherlich die Kriegserfahrungen. Als Regierungschef während der Schlacht von Verdun, dem Synonym für den Wahnsinn des totalen Krieges, war er von dem Bestreben geleitet, seinen französischen Landsleuten und der Welt ein solches Massentöten für alle Zukunft zu ersparen ${ }^{63}$. Dies machte ihn keineswegs zu einem Pazifisten, der den Krieg als Mittel der Politik generell ablehnte. Er wollte jedoch ein für alle Mal erreichen, daß die Sicherheit Frankreichs gewährleistet war, mithin eine internationale Ordnung existierte, die einen neuen bewaffneten Konflikt unmöglich machte. Dieses Bedürfnis wurde von einem Großteil der politischen Klasse Frankreichs geteilt. Dem Comité français de coopération européenne, das sich dem Ziel verschrieben hatte de ")développer la coopération des peuples de l'Europe dans le cadre et dans l'esprit de la Société des Nations « ${ }^{64}$, gehörte ein Großteil der Prominenz des Landes an. Als Ehrenpräsident dieser Vereinigung fungierte der Präsident der Republik, Gaston Doumergue, dem Ehrenkomitee gehörten unter anderem Poincaré, Briand, Caillaux, Herriot und Painlevé an. Zu den Mitgliedern gehörte - wie zur Union douanière - wiederum Seydoux. Briands besonderes europäisches Enga-

${ }^{60}$ Ibid.

${ }^{61}$ Siehe BARIÉTY, Briand, S. 134.

${ }^{62}$ Siehe KNIPPING, Locamo-Ära, S. 58.

${ }^{63}$ Siehe Jacques BARJETY, Aristide Briand. Les raisons d'un oubli, in: Antoine FLEURY, Lubor JiLEK (Hg.), Le Plan Briand d'Union fédérale européenne. Perspectives nationales et transnationales, avec documents. Actes du colloque tenu à Genève du 19 au 21 septembre 1991 , Bern u.a. 1998, S. 1-13, hier S. 6.

${ }^{64}$ »Comité français de coopération européenne« [Mai 1927], Fundort: AN 313 AP, 220. 
gement wurde auch daran deutlich, daß er sich in der Paneuropa-Union des österreichischen Grafen Richard Coudenhove-Kalergi engagierte, deren Ehrenpräsident er war ${ }^{65}$. Coudenhove-Kalergi forderte eine enge deutschfranzösische Kooperation, paneuropäische Abrüstung und Militärkontrolle sowie eine deutsch-polnische Verständigung und eine Zollunion, unter der Voraussetzung allerdings, daß zwischen Deutschland und Frankreich Gleichberechtigung herrsche und die Revision offen gehalten würde ${ }^{66}$. Die Ehrenpräsidentschaft Briands in dieser Organisation war deshalb bemerkenswert, weil Teile des Quai d'Orsay den Zielen der Paneuropa-Union kritisch gegenüber standen. Seydoux lehnte vor allem den Ausschluß Englands und Rußlands aus den Plänen Coudenhoves ab, denn dies führe "forcément à un groupement des Puissances dominé par l'Allemagne ${ }^{67}$, eine Kritik, die auch Berthelot teilte ${ }^{68}$.

Den Vorbehalten des Quai d'Orsay schloß sich die insgesamt noch kritischere Reichsregierung an. Bülow stellte fest: "Die große Schwäche der Coudenhove'schen Pläne lag von Anfang an darin, daß aus seiner Konzeption Paneuropas England und Rußland ausgeschlossen sein sollten ${ }^{69}$. Da der von Coudenhove geplante europäische Staatenverband wnichts anderes als ein Abklatsch des Völkerbunds ${ }^{70}$ sei, sei darüber hinaus zu befürchten, daß er zur Schwächung des Völkerbunds führen würde, was »vom deutschen Standpunkt durchaus unerwünscht wäre « ${ }^{71}$. Bülow riet auch deshalb zur Zurückhaltung, weil Coudenhove win den letzten Jahren in steigendem Maße französische Tendenzen zu unterstützen schien ${ }^{72}$ und empfahl, daß die Reichsregierung nicht offiziell an Veranstaltungen der Paneuropa-Union teilnehmen sollte, was dann auch so geschah.

Auch die Pläne Arnold Rechbergs für ein deutsch-französisches Militärbündnis - die sich im Grunde genommen in eine ähnliche Richtung bewegten, wie die Coudenhove-Kalergis - stießen in Frankreich auf eine wesentlich gröBere Resonanz als in Deutschland. Die Gründe für die vergleichsweise größere französische Akzeptanz und die deutsche Skepsis waren dabei stets die gleichen: In Frankreich erblickte man darin ein probates Mittel zur Erhöhung der

\footnotetext{
${ }^{65}$ Siehe Martin POSSELT, Die deutsch-französischen Beziehungen und der Briand-Plan im Spiegel der Zeitschrift Paneuropa, 1927-1930, in: Antoine FLEURY, Lubor JILEK (Hg.), Le Plan Briand d'Union fédérale européenne. Perspectives nationales et transnationales, avec documents. Actes du colloque tenu à Genève du 19 au 21 septembre 1991, Bern u.a. 1998, S. 31-51, hier S. 33.

${ }_{66}$ Siehe Reinhard FromMELT, Paneuropa oder Mitteleuropa. Einigungsbestrebungen im Kalkül deutscher Wirtschaft und Politik, Stuttgart 1977 (Schriftenreihe der VfZG, 34), S. $11-16$.

${ }^{67}$ Aufzeichnung Seydoux (4.3.1927), MAE PAAP 261, 37.

${ }^{68}$ Siehe Berthelot an Seydoux (5.4.1928), MAE PAAP 261, 42.

${ }^{69}$ Aufzeichnung Bülow (5.5.1930), PAAA R, 70104.

${ }^{70}$ Ibid.

71 Ibid.

${ }^{22}$ Ibid.
} 
eigenen Sicherheit, während in Deutschland vor allem bitter aufstieß, daß die Revisionsmöglichkeiten durch derlei weitgehende Verpflichtungen eingeschränkt würden.

Neben den sicherheitspolitischen Implikationen, die sich aus den verschiedenen Projekten für einen Zusammenschluß Europas, seien sie nun von Rechberg, Coudenhove-Kalergi oder anderen, ergaben, muß aber sicherlich auch das visionäre, idealistische Element gewürdigt werden, und hier ist das besondere Engagement Briands zu betonen.

Allerdings mußten die Ideen Briands für eine engere europäische Zusammenarbeit lange reifen, bis er sie mit seiner spektakulären Rede vor dem Völkerbund am 5. September 1929 vor der Welt verkündete. Als entscheidendes Ereignis für die Hinwendung Briands nach Europa sieht Bariéty hauptsächlich den für Frankreich verheerenden Verlauf der Washingtoner Flottenkonferenz von 1922: Nur durch einen Zusammenschluß Europas, resümierte der damalige französische Ministerpräsident, könne vermieden werden, daß Europa künftig zwischen den USA und Rußland zerrieben werde ${ }^{73}$. Praktische Konsequenzen hatte dies zunächst keine, denn Briand mußte, nachdem er von Präsident Millerand auf der Konferenz von Cannes öffentlich gemaßregelt worden war, die Regierung verlassen. Einen neuen Impuls gewann der Europa-Gedanke bei Briand nach dem Abschluß der Verträge von Locarno, weil diese nach französischer Lesart das Sicherheitsproblem noch keineswegs gelöst hatten. Vor der Kammer erklärte er Anfang 1926 die Notwendigkeit eines europäischen Föderalstaates nach Vorbild der USA ${ }^{74}$. In den Jahren 1927/1928 erwähnte er Europa weniger, was Bariéty jedoch weniger mit nachlassendem Interesse erklärt, sondern damit, daß Europa bereits "gemacht « wurde ${ }^{75}$ : Deutschland trat dem Völkerbund bei, die Abrüstungsverhandlungen begannen, und auch auf wirtschaftlichem Gebiet kam es durch die Internationale Rohstahlgemeinschaft und die Handelsvertragsverhandlungen zu einer Annäherung zwischen Deutschland und Frankreich. Gleichzeitig mußte Briand, nachdem Poincaré wieder Ratspräsident geworden und der Versuch einer deutsch-französischen "Gesamtlösung " in Thoiry nicht zuletzt am Widerstand des französischen Regierungschefs gescheitert war, vorsichtiger agieren. Ab 1928 erwachte jedoch erneut das Interesse Briands an einer stärkeren europäischen Kooperation ${ }^{76}$. Nachdem Frankreich seine Position in den Verhandlungen mit den USA, die zum späteren Kriegsächtungspakt führen sollten, nicht hatte durchsetzen können, wurde Briand erneut klar, daß aus sicherheitspolitischer Sicht nicht viel von Washington zu erwarten war ${ }^{77}$. Das Verhalten der USA in der Kriegs-

\footnotetext{
${ }^{73}$ Siehe BARIETY, Raisons d'un oubli, S. 6 f.

${ }^{74}$ Siehe ibid. S. 8.

75 „On , faisaitc l'Europe«, ibid. S. 9.

${ }^{76}$ Siehe HEYDE, Reparationen, S. 63.

${ }^{77}$ Siehe BARIÉTY, Pacte, S. 360.
} 
schuldenfrage und der Zollpolitik sowie in der Minderheitenfrage und anderen Probleme im Völkerbund stärkte sein Interesse an einer besseren europäischen Zusammenarbeit ${ }^{78}$.

Im März 1929 fertigte Jacques Rueff im Auftrag Légers eine Aufzeichnung an, »die als Urform des Europaplans gelten kann ${ }^{79}$ : Diese Aufzeichnung beinhaltete einen "pacte économique" der europäischen Regierungen, um die Stellung Europas in der Weltwirtschaft zu sichern. Ziel dieses Pakts sollte ein gemeinsamer Markt sein, wobei Einzelheiten den Beratungen von Experten vorbehalten werden sollten. Der neue, noch protektionistischere amerikanische Smoot-Harley-Zolltarif, der am 7. Mai 1929 in den Kongreß eingebracht wurde, und die zunehmende Zusammenarbeit zwischen deutschen und amerikanischen Unternehmen - zwischen General Motors und Opel bzw. General Electric und der AEG wurden Anfang 1929 Kooperationsabkommen unterzeichnet - verstärkten diese Forderungen noch ${ }^{80}$.

Im April 1929 gelangte an die Öffentlichkeit, daß Briand nach dem erfolgreichen Abschluß der Reparationsverhandlungen eine europäische Zollunion und den Ausbau der deutsch-französischen Industriekartelle anstrebe. Auch beim Völkerbund in Genf griff »[d]er Gedanke an die Schaffung eines 'Europäischen Zollvereins` [...] unzweifelhaft mehr und mehr um sich ${ }^{81}$.

Auf der Ratstagung des Völkerbunds im Juni 1929 in Madrid erkundigte sich Briand bei Stresemann, was dieser über die französischen Zollunionsvorstellungen dächte ${ }^{82}$. Aufgrund der antiamerikanischen Stoßrichtung der französischen Pläne verhielt sich der deutsche Außenminister jedoch zurückhaltend $^{83}$. Auch in Großbritannien herrschte wegen der Rolle der Dominions eine gewisse »Ratlosigkeit ${ }^{84}$, was die französischen Ideen anging. Aus der Reaktion seiner Gesprächspartner zog Briand zwei Konsequenzen: Zum einen rückte der Quai d'Orsay von seiner prononciert antiamerikanischen Linie $a b^{85}$, zum anderen lancierten Briand und andere französische Politiker die Europaideen verstärkt in der Öffentlichkeit ${ }^{86}$, wobei sich auch die Paneuropa-Bewegung an dieser Aktion beteiligte ${ }^{87}$.

\footnotetext{
${ }^{78}$ Siehe KNIPPING, Locamo-Ära, S. 86.

${ }^{79}$ Siehe HEYDE, Reparationen, S. 63

${ }^{80}$ Siehe KNIPPING, Locamo-Ära, S. 86.

${ }^{81}$ Dufour an Köpke (8.4.1929), ADAP B XI, Nr. 162.

${ }^{82}$ Siehe Aufzeichnung Schmidt (11.6.1929), ADAP B XU, Nr. 19.

${ }^{83}$ Siehe Peter KRÜGER, Der abgebrochene Dialog. Die deutschen Reaktionen auf die Europavorstellungen Briands 1929, in: Antoine FleURY, Lubor JILEK (Hg.), Le Plan Briand d'Union fédérale européenne. Perspectives nationales et transnationales, avec documents. Actes du colloque tenu à Genève du 19 au 21 septembre 1991, Bern u.a. 1998, S. 289-306, hier S. 304.

${ }^{84}$ KNIPPING, Locarno-Ära, S. 88.

${ }^{85}$ Siehe Hoesch an AA 12.7.1929), ADAP B XII, Nr. 87.

${ }^{86}$ Siehe BARIETY, Raisons d'un oubli, S. 12f.

${ }^{87}$ Siehe POSSELT, Paneuropa, S. 42.
} 
In Deutschland blieb die Reaktion darauf weiterhin "skeptisch abwartend ${ }^{88}$. Zwar wurde die wirtschaftliche Zusammenarbeit begrüßt, allerdings vermutete man weiterhin eine antiamerikanische Spitze hinter den französischen Plänen ${ }^{89}$. Auf völlige Ablehnung stieß jedoch die politische Komponente des Briand-Projekts, da sie nach Auffassung des AA vor allem dazu dienen sollte, den Status quo in Europa zu zementieren und die französische Sonderstellung in Europa zu sichern ${ }^{90}$.

Briand ließ sich von diesen kühlen Reaktionen des Auslands jedoch nicht beirren und auch die diesbezüglichen Warnungen Légers und Massiglis schlug er in den Wind ${ }^{91}$. Die neue Labour-Regierung in England, die er für aufgeschlossener gegenüber seinen Ideen hielt als die Tories ${ }^{92}$, und der Rücktritt seines wichtigen potentiellen innenpolitischen Widersachers Poincarée ${ }^{93}$ stimmten ihn optimistisch. Nachdem durch die Haager Konferenz auch endlich das leidige Reparationsproblem und die Frage der Rheinlandräumung erledigt waren, hielt Briand den Moment für seine Europainitiative für gekommen, zumal er durch Indiskretionen der Presse in Zugzwang geriet, konkrete Vorschläge zu unterbreiten ${ }^{94}$. Am 5. September 1929 hielt Briand schließlich seine Rede vor der Vollversammlung des Völkerbunds, in der er die Schaffung eines 》lien fédéral ${ }^{95} \mathrm{zwischen} \mathrm{den} \mathrm{europäischen} \mathrm{Staaten} \mathrm{forderte.}$

Vor allem Deutschland und Großbritannien blieben die Vorschläge zu vage, sie forderten Konkretisierungen ${ }^{96}$. Gleichzeitig begannen beide Länder, die den Vorschlägen Briands skeptisch bis ablehnend gegenüberstanden, den französischen Vorstellungen den Wind aus den Segeln zu nehmen: Stresemann betonte in seiner Antwort vor dem Völkerbund vor allem die wirtschaftlichen Aspekte des briandschen Vorschlags und verband dies mit der Forderung, daß sich die europäische Kooperation nicht gegen die USA richten dürfe ${ }^{97}$. Die Friedenssicherung sah er vor allem in einem Ausbau der Schiedsgerichtsbarkeit, der Einbindung des Briand-Kellogg-Pakts in die Satzung des Völker-

\footnotetext{
${ }^{88}$ KNIPPING, Locamo-Ära, S. 88.

${ }^{89}$ Siehe ibid.

${ }^{90}$ Siehe Aufzeichnung Schubert (1.8.1929), ADAP B XII, Nr. 138.

${ }^{91}$ Siehe BARIETY, Raisons d'un oubli, S. 13.

92 Siehe Aufzeichnung Schmidt (11.6.1929), ADAP B XII, Nr. 19.

${ }^{93}$ Siehe BARIETY, Raisons d'un oubli, S. 12.

${ }^{94}$ Siehe ibid. S. $12 \mathrm{f}$.

${ }^{95}$ Die Rede Briands ist teilweise abgedruckt in: Schulthess' Europäischer Geschichtskalender, N.F., 45. Jg. (1929), S. 539-541.

${ }^{96}$ Siehe HEYDE, Reparationen, S. 63.

${ }^{97}$ Rede Stresemanns: BERNHARD, Stresemann: Vermächtnis, Bd. 3, S. 570-580, insbesondere S. 577f.; siehe auch: Martin VOGT, Die deutsche Haltung zum Briand-Plan im Sommer 1930. Hintergruinde und politisches Umfeld der Europapolitik des Kabinetts Brüning, in: Antoine FLEURY, Lubor JILEK (Hg.), Le Plan Briand d'Union fédérale européenne. Perspectives nationales et transnationales, avec documents. Actes du colloque tenu à Genève du 19 au 21 septembre 1991, Bem u.a. 1998, S. 305-329, hier S. 311.
} 
bunds und der allgemeinen Abrüstung begründet ${ }^{98}$, wofür seines Erachtens keineswegs ein neues europäisches Organ notwendig wäre. Stresemann versuchte so, besonders die möglichen politischen Implikationen des Vorschlags zu verhindern. Großbritannien, das eine engere europäische Wirtschaftskooperation wegen seines Präferenzsystems für das Empire ablehnte, schlug dagegen einen Zollfrieden für zwei Jahre vor, damit während dieses Zeitraums über Zollreduzierungen verhandelt werden könnte ${ }^{99}$. Der Charme dieses Vorschlags lag darin, daß er konkret war, während die französische Initiative unklar geblieben war. Außerdem richtete sich auch dieser Plan weniger offensichtlich gegen die USA.

Nichtsdestotrotz wurde die französische Regierung von der Vollversammlung aufgefordert, ein Memorandum in der Europafrage zu entwerfen, das von den Regierungen kommentiert werden sollte. Im Winter 1929/1930 beschäftigten sich vor allem Massigli und Léger ${ }^{100}$ mit der Ausarbeitung des französischen Europamemorandums, das am 17. Mai 1930 - dem Tag, an dem die Räumung der letzten Besatzungszone im Rheinland angekündigt wurde - den Regierungen der europäischen Mitgliedsstaaten des Völkerbunds übergeben wurde ${ }^{101}$.

Das Memorandum gliederte sich in fünf Teile. Im ersten Teil des Memorandums wurden allgemeine Grundsätze der europäischen Einigung dargelegt. Dort wurde betont, daß die europäische Kooperation nur in Zusammenarbeit mit dem Völkerbund erfolgen könne. Außerdem wurde festgestellt, daß sich die engere europäische Wirtschaftskooperation nicht gegen andere Staaten richte und auch an eine Einschränkung der Souveränität der Staaten nicht gedacht sei. In einem Elementarvertrag sollten möglichst knapp die Ziele und die Organisation des europäischen Zusammenschlusses sowie das Verhältnis zum Völkerbund geregelt werden. Der zweite Abschnitt befaßte sich mit der Organisation der europäischen Zusammenarbeit. Neben der Conférence européenne, in die alle Staaten Vertreter entsenden sollten, sollte es noch einen politischen Ausschuß geben sowie ein Sekretariat zur Koordination der Arbeit der Konferenz und des Rates. Im Grunde genommen handelte es sich um den gleichen Aufbau wie beim Völkerbund ${ }^{102}$. Im dritten Teil wurden Leitlinien für

\footnotetext{
${ }^{98}$ Siehe BERNHARD, Stresemann: Vermächtnis, Bd. 3, S. 572-574.

${ }^{99}$ Zum folgenden siehe SCHULZ, Wirtschaftsordnung, S. 183-185.

${ }^{100}$ Siehe Hoesch an AA (20.6.1930), ADAP B XV, Nr. 90.

101 Deutscher Text: Schulthess' Europäischer Geschichtskalender, N.F., 46. Jg. (1930), S. 460-468; Französischer Text in: Antoine FleURY, Lubor JILEK (Hg.), Le Plan Briand d'Union fédérale européenne. Perspectives nationales et transnationales, avec documents. Actes du colloque tenu à Genève du 19 au 21 septembre 1991, Bern u.a. 1998, Anhang I. Zum folgenden siehe ibid.

${ }^{102}$ Siehe Antoine FleURY, Avant-propos, in: DERS., Lubor JILEK (Hg.), Le Plan Briand d'Union fédérale européenne. Perspectives nationales et transnationales, avec documents.
} 
die europäische Zusammenarbeit vorgeschlagen. Wichtigste Bestimmung war die "[a]llgemeine Unterordnung des Wirtschaftsproblems unter das politische Problem $"{ }^{103}$, weil auch die wirtschaftliche Einigung "streng durch die Sicherheitsfrage bestimmt wird ${ }^{104}$. Sonst könne nicht gewährleistet werden, daß die schwächeren Staaten nicht der Beherrschung durch die stärkeren ausgesetzt würden. Die politische Zusammenarbeit sollte, bei Beibehaltung der Souveränität der Staaten, durch den Ausbau des Schiedsvertragswesens und die Ausdehnung der Locarno-Garantien auf ganz Europa erreicht werden. Verschiedene lokale Sicherheitssysteme (wie beispielsweise das von Locarno) sollten außerdem zu einem gesamteuropäischen System zusammengefaßt werden. Im wirtschaftlichen Bereich wurden die Errichtung eines gemeinsamen Marktes sowie die Vereinfachung des Güter-, Kapital- und Personenaustauschs »lediglich unter dem Vorbehalt der Bedürfnisse der nationalen Verteidigung in jedem Staate ${ }^{105}$ als Leitlinie formuliert. Der vierte Teil des Memorandums umfaßte eine Zusammenfassung der noch offenen Fragen bezüglich der künftigen Gestalt Europas. Dabei ging es unter anderem um die genaue Festlegung der Bereiche der europäischen Zusammenarbeit, wie beispielsweise die Umsetzung des Programms der Weltwirtschaftskonferenz, die Kartellkontrolle, gemeinsame Infrastrukturmaßnahmen oder die Förderung unterentwickelter Gebiete, die geistige Zusammenarbeit und den Ausbau interparlamentarischer Beziehungen. Nach Auffassung der französischen Regierung waren die Bestimmung der Methoden der europäischen Zusammenarbeit und des Verhältnisses des zu schaffenden europäischen Gebildes zu außereuropäischen Staaten weitere wesentliche noch $z u$ lösende Probleme. Im abschließenden Teil betonte die französische Regierung, daß es sich lediglich um Vorschläge handele, die langfristig zur Verbesserung der Lage in Europa beitragen sollten. Mit keinem Wort wurde im übrigen der Versailler Vertrag erwähnt.

Das französische Europamemorandum stand ganz in der Tradition der französischen Außenpolitik der 1920er Jahre: In ihm wurde das französische Bestreben sichtbar, die durch den Versailler Vertrag geschaffene europäische Ordnung zu stabilisieren und die immer noch als prekär empfundene Sicherheitslage Frankreichs zu verbessern. Besonders wurde dies an der Unterordnung wirtschaftlicher Fragestellungen unter vor allem sicherheitspolitische Aspekte deutlich. Die Aufnahme eines wirtschaftlichen Programms an sich stellte aber ein enormes Entgegenkommen der französischen Regierung dar. Sicherheitspolitisch bedeutete der französische Europaplan im Grunde genommen die Wiederanknüpfung an das Genfer Protokoll, diesmal auf regiona-

Actes du colloque tenu à Genève du 19 au 21 septembre 1991, Bern u.a. 1998, S. I-XVI, hier S. XII.

${ }^{103}$ Europamemorandum.

${ }^{104}$ Ibid.

${ }^{105}$ Ibid. 
ler, europäischer Ebene. Die Schlichtungsmechanismen sollten ausgebaut und eine Art Superlocarno für ganz Europa sollte geschaffen werden. Neu indes war, daß im Gegenzug für die Erfüllung der französischen Sicherheitswünsche eine verstärkte wirtschaftliche Zusammenarbeit angeboten wurde, die nach herrschender Auffassung wohl vor allem Deutschland zugute gekommen wä$\mathrm{re}^{106}$. Und war es nicht Stresemann gewesen, der seit 1925 von einer Zollunion gesprochen hatte, und diese Forderung in seiner letzten großen Rede wiederholt hatte ${ }^{107}$ ? Im Grunde genommen - dies war allerdings nur die deutschlandpolitische Ebene des Briand-Plans - hatte die französische Regierung der Reichsregierung einen Deal vorgeschlagen: Anerkennung der politischen europäischen Nachkriegsordnung gegen Erfüllung der wirtschaftlichen Forderungen Deutschlands. Dabei war nicht völlig auszuschließen, daß man auch den deutschen politischen Forderungen in den Verhandlungen entgegengekommen wäre, denn der Vorschlag, wie ihn Frankreich am 17. Mai 1930 vorgelegt hatte, stellte ein erstes Verhandlungsangebot dar, nicht das letzte Wort.

In Deutschland wurden natürlich die sicherheitspolitischen Implikationen erkannt. Aus dem Text des Memorandums zitierend, stellte Staatssekretär Schubert fest:

`Ein ständiges System vertraglich festgelegter Solidarität ‘ [Herv. i.O.], wir wissen, was diese Worte im Sprachschatz der französischen Politik bedeuten. Sie bedeuten den Wunsch, durch immer neue Abmachungen den durch die Friedensverträge i.J. 1919 geschaffenen Zustand Europas zu stabilisieren und durch den Aufbau eines Sanktionssystems gegen den Friedensbrecher sicherzustellen ${ }^{108}$.

Hoesch kam zu ähnlichen Schlüssen: »Paul-Boncour und andere Persönlichkeiten haben ganz offen ihrer Meinung Ausdruck gegeben, daß das Briandsche Projekt einen bedeutsamen und vielleicht den letzten möglichen Versuch darstelle, ein System der Friedenssicherung durch gegenseitige Unterstützung im Sinne des Genfer Protokolls doch noch auf die Füße zu stellen « ${ }^{109}$. Schubert, der bald als Staatssekretär abgelöst und als Botschafter nach Rom "abgeschoben « werden sollte, und Hoesch gehörten allerdings zu den wenigen, die auf deutscher Seite auch die positiven Ansätze des Europamemorandums zu würdigen wuBten. Der deutsche Botschafter in Paris sah in Briands Vorschlag keineswegs ein $" Z$ Weckmanöver ${ }^{110}$, Frankreich sei vielmehr ernsthaft auch am wirtschaftlichen Zusammenschluß Europas interessiert. Er äußerte jedoch Zweifel, ob das von Frankreich vorgeschlagene Verfahren, zuerst den politi-

\footnotetext{
${ }^{106}$ Siehe Aufzeichnung ohne Unterschrift [1924], BArch R 3101, 20458.

${ }^{107}$ Vgl. Margerie an Quai d'Orsay (29.8.1925), MAE 1918-1929 Z (Europe) Allemagne, 524; BERNHARD, Stresemann: Vermächtnis, Bd. 3, S. 570-580.

${ }^{108}$ Schubert an Reichskanzlei (26.5.1930), AdR Brüning III Bd. 1, Nr. 40.

${ }^{109}$ Hoesch an AA (20.6.1930), ADAP B XV, Nr. 90.

${ }^{110}$ Ibid.
} 
schen Rahmen und dann erst die wirtschaftliche Kooperation festzulegen, aufgrund der akuten Wirtschaftskrise in Europa praktikabel sei ${ }^{111}$. Auch Schubert sah vor allem in den wirtschaftlichen Vorschlägen des französischen Memorandums einen "guten Kern ${ }^{112}$, wenngleich er sich dafür aussprach, daß ein gegen die USA und die Sowjetunion gerichteter Kontinentalblock verhindert werden müsse und der Völkerbund nicht geschwächt werden dürfe ${ }^{113}$. Bülow, der kommende starke Mann im AA, lehnte dagegen den französischen Europaplan $a b^{114}$. Ohne die wirtschaftlichen Aspekte und den Vorschlagscharakter des Memorandums ausreichend zu würdigen, kam er zu dem Ergebnis, daß der Briand-Plan hauptsächlich der Sicherung der französischen Vormachtstellung und des Status quo diene. Deutschland würde damit Frankreich dauerhaft untergeordnet $\operatorname{sein}^{115}$. Er stellte fest: »Diese Stellungnahme führe dazu, daß man eigentlich den politischen Teil des Memorandums restlos ablehnen müsse, infolgedessen auch der von Briand gewünschten Unterordnung der Wirtschaft unter die Politik nicht zustimmen könne « ${ }^{116}$. Dieser Auffassung schloß sich Julius Curtius, seit dem Tod Stresemanns am 3. Oktober 1929 neuer deutscher Außenminister, weitgehend $a^{117}$. Curtius erkannte dabei eine entscheidende Schwachstelle des französischen Vorschlags, daß nämlich die politischen Fragen - wie die Abrüstungsverhandlungen und der Kriegsächtungspakt gezeigt hätten - nicht ohne die Vereinigten Staaten und die Sowjetunion gelöst werden könnten. Es ist an verschiedenen Stellen darauf hingewiesen worden, daß - wegen der prekären machtpolitischen Situation zwischen Deutschland und Frankreich - ein stabilisierendes Element, wie eine aktive Einflußnahme Großbritanniens und der USA, notwendig gewesen wäre. Weder bei Curtius noch bei Bülow wurde diese Erkenntnis allerdings in aktive Politik umgesetzt, sondern als ein allein negatives Argument zur Ablehnung des französischen Vorschlags verwandt.

$\mathrm{Daß}$ der französische Köder - die wirtschaftliche Kooperation - von vielen in Deutschland verschmäht wurde, lag vor allem daran, daß sich die deutsche Außenwirtschaftspolitik, wie bereits dargelegt wurde, vor allem auf Druck des Reichsernährungsministeriums ${ }^{118}$ ab $1929 / 1930$ von ihrer liberalen Grundhal-

${ }^{111}$ Siehe ibid.

${ }^{112}$ Aufzeichnung Schubert (28.5.1930), ADAP B XV, Nr. 52.

${ }^{113}$ Siehe ibid.

${ }^{114}$ Siehe VOGT, Deutsche Haltung, S. 309; Aufzeichnung Bülow (21.5.1930), ADAP B XV, Nr. 39.

${ }^{115}$ Siehe VoGT, Deutsche Haltung, S. 310.

${ }^{116}$ Aufzeichnung Planck (19.6.1930), AdR Brüning I/II Bd. 1, Nr. 51, ebenfalls abgedruckt in: Reinhard OPITZ (Hg.), Europastrategien des deutschen Kapitals, 1900-1945, Köln 1977, Nr. 65.

${ }^{117}$ Siehe Aufzeichnung Bülow (11.6.1930), ADAP B XV, Nr. 71, siehe auch zum folgenden.

${ }^{118}$ Aufzeichnung Planck (19.6.1930), AdR Brüning I/II Bd. 1, Nr. 51. Zu den zunehmend protektionistischen Forderungen aus der Schwerindustrie und der Landwirtschaft siehe FROMMELT, Paneuropa, S. 78-80. 
tung zunehmend löste und protektionistischer wurde. Wie am Vorschlag Curtius' für eine deutsch-österreichische Zollunion vom März 1931 deutlich wurde, wandte sich Deutschland vom Freihandel ab, hin zu einem mitteleuropäischen Wirtschaftsblock unter deutscher Kontrolle ${ }^{119}$.

Auffällig ist auch, daß deutsche Diplomaten und Politiker argumentierten, ein Eingehen auf die französischen Vorschläge würde die deutschen Revisionsmöglichkeiten einschränken ${ }^{120}$. Gleichzeitig entwickelte die deutsche Seite aber keine genauen Vorstellungen darüber, welches Revisionsziel als nächstes angestrebt werden sollte, ja welche Revisionsziele realistischerweise überhaupt erreichbar waren. Die französische Initiative hätte von Deutschland genutzt werden können, zumindest die Spielräume für die zukünftig deutsche Revisionspolitik auszuloten und unter Umständen auch zu konkreten Revisionsschritten zu kommen. Allerdings muß man sagen, daß die deutschen Revisionsmöglichkeiten - was Frankreich anging - begrenzt waren ${ }^{121}$ : Die Rückgabe des Korridors, nicht jedoch Oberschlesiens, schien möglich. Den Anschluß Österreichs lehnte Frankreich ab, und das militärische Übergewicht gegenüber Deutschland mußte erhalten bleiben. Daß in Deutschland, nachdem wichtige Revisionsziele bis Ende der 1920er Jahre zweifelsohne erreicht worden waren - die Freigabe des Rheinlandes, eine Verringerung der Reparationen, die wirtschaftliche und politische (nicht jedoch militärische) Gleichberechtigung -, keine weiteren konkreten Revisionsziele mehr formuliert wurden, hatte möglicherweise einen ganz einfachen Grund: Es fehlte die Bereitschaft, anzuerkennen, daß viele Revisionsziele durch die Politik der friedlichen Verständigung sich nicht würden erreichen lassen. Indem die Revisionspolitik auf eine vage Zukunft verschoben wurde, konnte man an der Fiktion festhalten, weiterhin die Revision der deutschen Ostgrenzen zu betreiben. Dies war vor allem auch im Hinblick auf die deutsche Öffentlichkeit nötig, denn die vorzeitige Rückgabe des Rheinlandes hatte nicht etwa zur Dämpfung, sondern $\mathrm{zu}$ einem neuen Aufflammen des Revisionismus gefuhrrt ${ }^{122}$. Da das politische System zunehmend in die Krise geriet, wofür der Rücktritt der Regierung Müller und der Beginn des Präsidialregimes unter Brüning den augenscheinlichsten Beleg darstellte, fehlte vielleicht die Kraft zu dieser Einsicht ${ }^{123}$. Gleichzeitig manövrierte sich die deutsche Politik aber auch in die Handlungsunfähigkeit, weil sie sich einer realistischen Einschätzung verweigerte und auf Abwarten setzte.

\footnotetext{
${ }^{119}$ Siehe Posselt, Paneuropa, S. 49.

${ }^{120}$ Vgl. beispielsweise: Aufzeichnung Bülow (21.5.1930), ADAP B XV, Nr. 39; Aufzeichnung Planck (19.6.1930), AdR Briuning VII Bd. 1, Nr. 51; Schubert an Reichskanzlei (26.5.1930), AdR Briuning I/II Bd. 1, Nr. 40; Hammerstein an AA (12.7.1930), ADAP B XV, Nr. 76.

${ }^{121}$ Siehe WURM, Deutschlands Rolle, S. 165, 169.

122 Siehe VOGT, Deutsche Haltung, S. 319.

${ }^{123}$ Vgl. WRIGHT, Stresemann, S. 454-457.
} 
Die deutsche Antwort auf das französische Memorandum, die am 15. Juli 1930 übergeben wurde ${ }^{124}$, war dem Ton nach zwar zustimmend, enthielt aber so viele Vorbehalte, daß sie einer Ablehnung gleichkam. Zwar wurde ein gemeinsames europäisches Vorgehen in der Zollpolitik befürwortet, allerdings wurde festgestellt, daß die europäische Zusammenarbeit in dieser Frage nicht gegen andere Länder gerichtet sein dürfe. Die Unterordnung der wirtschaftlichen unter die politischen Fragen lehnte Deutschland ab und forderte statt dessen Gleichberechtigung auch auf militärischem Gebiet. Die deutsche Antwort auf das französische Europamemorandum war in zweifacher Hinsicht eine verpaßte Chance. Einerseits wurde versäumt, die zugegebenermaßen flachen Wasser deutscher Revisionsmöglichkeiten auszuloten. Andererseits unterblieb eine realistische Bewertung der deutschen Außenpolitik, ihrer Ziele ebenso wie ihrer Methoden. In der Tat, Deutschland war nicht bereit, »die ihm zukommende Ordnungsfunktion einer Großmacht zu erfüllen und seine Revisionsforderungen übernationalen Interessen unterzuordnen ${ }^{125}$ und versäumte es, eine "geschlossene Konzeption für eine künftige europäische Ordnung ${ }^{126} \mathrm{zu}$ entwickeln.

Es wäre indes falsch, allein Deutschland für das Scheitern des französischen Europaplanes verantwortlich zu machen. Auch Italien, die Niederlande, Belgien und Polen äußerten sich zu den Vorschlägen skeptisch ${ }^{127}$. Kritik kam auBerdem aus dem Völkerbund selbst, der durch die Pläne Briands seine Marginalisierung befürchtete ${ }^{128}$.

Ausschlaggebend dürfte - wie bei vielen anderen wichtigen Entscheidungen bezüglich der deutsch-französischen Beziehungen - die Ablehnung durch die englische Regierung gewesen sein ${ }^{129}$. Der englische Außenminister Henderson warnte vor der Regionalisierung des Völkerbunds ${ }^{130}$ und der antiamerikanischen Spitze des französischen Vorschlags ${ }^{131}$. Großbritannien wandte sich allerdings auch deshalb gegen den Europaplan, weil es befürchtete, neue politi-

\footnotetext{
${ }^{124}$ Text: Schulthess' Europäischer Geschichtskalender, N.F., 46. Jg. (1930), S. 469-472.

${ }^{125}$ BERG, deutsche Locarnopolitik, S. 269.

${ }^{126}$ KRÜGER, Außenpolitik, S. 7.

${ }^{127}$ Siehe Ministerbesprechung (5.7.1930), AdR Brüning I/I Bd. 1, Nr. 65. Eine Zusammenfassung der Antworten findet sich in: Aufzeichnung ohne Unterschrift (30.8.1930), BArch R $2501,6317$.

${ }^{128}$ Siehe Marie-Renée Mouton, La Société des Nations et le Plan Briand d'Union europénne, in: Antoine FleURY, Lubor JILEK (Hg.), Le Plan Briand d'Union fédérale européenne. Perspectives nationales et transnationales, avec documents. Actes du colloque tenu à Genève du 19 au 21 septembre 1991, Bern u.a. 1998, S. 235-255, hier S. 238f.

${ }^{129}$ Zusammenfassend hierzu: Andrea Bosco, The British Foreign Office and the Briand Plan, in: Antoine FLeURY, Lubor JiLEK (Hg.), Le Plan Briand d'Union fédérale européenne. Perspectives nationales et transnationales, avec documents. Actes du colloque tenu à Genève du 19 au 21 septembre 1991, Bern u.a. 1998, S. 347-358.

${ }^{130}$ Siehe Aufzeichnung ohne Unterschrift (9.9.1929), ADAP B XIII, Nr. 9

${ }^{131}$ Siehe Aufzeichnung ohne Unterschrift (30.8.1930), BArch R 2501, 6317.
} 
sche Garantien und mehr Verantwortung in Europa übernehmen zu müssen ${ }^{132}$. Allein Churchill äußerte sich positiv ${ }^{133}$.

Das Scheitern des Europaplans lag teilweise darin begründet, daß die Ideen Briands zu visionär waren, besonders in einer Zeit, in der die nationalen Egoismen - aufgrund der Weltwirtschaftskrise und der sozialen Spannungen, die daraus erwuchsen - zunahmen ${ }^{134}$. Problematisch war auch, daß in dem französischen Memorandum das Schwergewicht auf die politischen, nicht aber auf die viel drängenderen wirtschaftlichen Fragen gelegt wurde ${ }^{135}$. Dies hatte allerdings auch innenpolitische Gründe, denn der neue französische Regierungschef Tardieu forderte die stärkere Berücksichtigung sicherheitspolitischer Aspekte und war protektionistischer eingestellt ${ }^{136}$. Für das Schicksal des Briand-Plans war jedoch entscheidend, daß die französische Außenwirtschaftspolitik in sich widersprüchlich blieb. Stellte der Briand-Plan zwar einerseits die enge wirtschaftliche Zusammenarbeit in Europa in Aussicht, weigerte sich Frankreich andererseits hartnäckig, konkret am Abbau von Wirtschaftshemmnissen und Zollreduzierungen mitzuwirken, wobei sich die französische Vorsicht vor allem aus der Angst vor der deutschen Wirtschaftsmacht erklärte ${ }^{137}$. Für diese Widersprüche waren vor allem innerfranzösische Meinungsunterschiede ausschlaggebend. Während der Quai d'Orsay weiterhin liberal eingestellt war, lehnten das Agrarministerium, aber auch Ratspräsident Tardieu, die freihändlerische Politik ab. Auch von den französischen Wirtschaftsverbänden sprach sich nur einer, das Comité d'action économique et douanière, vorbehaltlos für eine liberale Außenhandelspolitik aus ${ }^{138}$.

Briands Europainitiative war ein ähnliches Schicksal beschieden wie so vielen anderen Plänen zur Neuordnung der internationalen Beziehungen: Auf britische und Schweizer Initiative setzte der Völkerbund am 17. September 1930 eine Studienkommission zu diesem Thema ein. Diese tagte bis 1932 mehrmals und kam zum letzten Mal am 1. Oktober 1937 zusammen, ohne je offiziell aufgelöst worden zu $\operatorname{sein}^{139}$.

Um auf die Ausgangsfrage dieses Kapitels zurückzukommen - nämlich warum die Modernisierung der Außenpolitik scheiterte -, läßt sich also im Hinblick auf das Schicksal des französischen Europamemorandums sagen: Die Fortsetzung einer modernen Außenpolitik scheiterte, weil in der Situation des Jahres 1929/1930 vor allem Deutschland versäumte, ein neues außenpoliti-

${ }^{132}$ Siehe Schubert an Reichskanzlei (26.5.1930), AdR Brüning V/II Bd. 1, Nr. 40; KRÜGER, Schwierigkeit, S. 128.

${ }^{133}$ Siehe Ministerbesprechung (5.7.1930), AdR Brüning I/I Bd. 1, Nr. 65.

${ }^{134}$ Siehe FLEURY, Avant-propos, S. XIV.

${ }^{135}$ Siehe ibid. S. XV.

${ }^{136}$ Siehe BUSSIËRE, Aspects économiques, S. 88; BADEL, Trêve douanière, S. 153, $159 f$.

${ }^{137}$ Siehe BOYCE, Limits, S. 114.

${ }^{138}$ Siehe BADEL, Trêve douanière, S. 150f., 159.

${ }^{139}$ Siehe PFEIL, Völkerbund, S. 99. 
sches Konzept mit modernen Zielsetzungen zu entwickeln. Allerdings standen auch der Briand-Plan und die tatsächlich von Frankreich verfolgte Politik faktisch in einem nicht unerheblichen Widerspruch zueinander. Der Briand-Plan behinderte aber auch deshalb die Modernisierung der Außenpolitik, weil durch ihn die Komplexität der internationalen Beziehungen, in einer Phase, als diese ohnehin schon labil waren, erhöht, und so eine Lösung erschwert wurde. In Kapitel 3 wurde dargelegt, daß das Reparationsproblem erst dann gelöst werden konnte, als durch das Vorgehen im Dawes-Plan die Reparations- von der Sicherheitsfrage abgetrennt worden war. Dies verringerte die bis dahin herrschende Komplexität der außenpolitischen Probleme, wodurch die Regelung des Reparationsproblems erst ermöglich wurde. Die Unterordnung der wirtschaftlichen Aspekte unter die Sicherheitspolitik im französischen Europamemorandum bewirkte genau das Gegenteil: Die Verquickung von beiden Problemen erhöhte die Komplexität der internationalen Beziehungen wieder. Es war gerade dieser Zusammenhang, der der deutschen Regierung die Ablehnung des französischen Memorandums erleichterte.

Es gab jedoch noch weitere Gründe, die die Krise der modernen Außenpolitik zu diesem Zeitpunkt verstärkten. In diesem Zusammenhang sind vor allem personenbezogene Faktoren zu nennen. Besonders hervorzuheben ist dabei sicherlich der Tod Stresemanns am 3. Oktober 1929. Sein Nachfolger Curtius lenkte "teils aus persönlicher Überzeugung, teils unter innerpolitischem Druck, die deutsche Außenpolitik in eine stärker national geprägte Richtung ${ }^{140}$. Die aufgrund persönlicher und politischer Differenzen zwischen ihm und Schubert erfolgte Ablösung des Staatssekretärs und die Ernennung Bülows zu Schuberts Nachfolger leitete ab 1930 die Verstärkung der Revisionspolitik und die Wendung nach Südost- und Ostmitteleuropa ein ${ }^{141}$. Das deutsch-österreichischen Zollunionsprojekt vom März 1931 markierte diesen Kurswechsel und beendete die Verständigungspolitik ${ }^{142}$. Zeitgleich mit den personellen Veränderungen in der deutschen Diplomatie vollzog sich der Rückgang des Einflusses von Briand auf die französische Außenpolitik. Briand blieb zwar nach dem Scheitern seines letzten Kabinetts am 22. Oktober 1929 Außenminister, Tardieu nahm aber zunehmend das Heft in die Hand ${ }^{143}$. Der neue französische Ratspräsident beurteilte die Verständigungspolitik skeptischer, beharrte stärker auf französischen Positionen und setzte - vor allem, nachdem sich das französisch-britische Verhältnis mit dem Amtsantritt der Labour-Regierung merklich abgekühlt hatte - auf eine unabhängigere Politik ${ }^{144}$. Der Personal- und Politikwechsel in Deutschland und

\footnotetext{
${ }^{140}$ KNIPPING, Locamo-Ära, S. 94; KRÜGER, Schwierigkeit, S. $129 f$.

${ }^{141}$ Siehe VOGT, Deutsche Haltung, S. 315.

${ }^{142}$ Siehe JACOBSON, Locarno Diplomacy, S. 356.

${ }^{143}$ Siehe ibid. S. 353; KNIPPING, Locarno-Ära, S. 95.

${ }^{144}$ Siehe KNIPPING, Locarno-Ära, S. 95.
} 
Frankreich fand seine Entsprechung auch in Großbritannien, wo der seit Juni 1929 amtierende Premier MacDonald von der profranzösischen Politik Chamberlains abrückte und zur klassischen Gleichgewichtspolitik zurückkehrte $^{145}$. Gerade in Zeiten des fehlenden politischen Konsenses ist mit dem Wechsel des Personals oft auch ein Politikwechsel verbunden. Dieser Austausch des politischen Personals betraf allerdings nicht nur die Außenpolitik. Ende der 1920er Jahre schien vielmehr ein tiefgehender Generationswechsel stattzufinden, der Personen in Führungspositionen brachte, die während des Ersten Weltkriegs in der Regel noch keine leitenden Funktionen innegehabt hatten und deshalb dieses epochale Ereignis aus einer anderen Perspektive wahrnahmen und andere Konsequenzen daraus zogen. Der Tod Fochs beispielsweise, ebenfalls im Jahre 1929, zementierte erst den radikalen Wechsel in der französischen Militärdoktrin von der Offensive zur Defensive. Solange der Generalissimus noch lebte, war eine Sicherheitspolitik, die allein auf die Befestigung der Ostgrenze abzielte, nur schwer möglich ${ }^{146}$. Im großen Stil wurde der Bau der Maginot-Linie erst nach seinem Tod in Angriff genommen.

Das relativ gute persönliche Verhältnis zwischen den "Großen Drei« der Locarno-Politik, Stresemann, Briand und Chamberlain, hatte allerdings auch Nachteile für die Modernisierung der Außenpolitik gehabt. Thre "Genfer Teeparties« hatten zwar dazu geführt, daß viele Probleme informell gelöst wurden, verhinderten bzw. verzögerten aber durch die Umgehung der Völkerbundsgremien auch den Aufbau internationaler Konfliktregelungsmechanismen, was übrigens schon von Zeitgenossen kritisiert wurde ${ }^{147}$. Neben der sichtbaren Einigkeit der »Großen Drei« bestanden die verschiedenen nationalen Interessen und Konflikte aber weiter und konnten deshalb auch in der Phase der relativ guten Beziehungen zwischen den drei Staaten nicht ausgeräumt werden ${ }^{148}$. Der Abgang Stresemanns, Briands und Chamberlains hinterließ auf der Ebene der multilateralen Diplomatie ein Vakuum, das paradoxerweise erst durch die überragende Rolle dieser drei entstanden war.

Nicht nur konzeptionelle und personale Faktoren trugen jedoch zum Scheitern der Modernisierung der Außenpolitik bei, sondern auch die internationalen Rahmenbedingungen. Wie bereits mehrfach angeklungen ist, war das deutsch-französische Verhältnis sehr labil und hätte dringend der Stabilisierung durch Dritte, vor allem durch die Vereinigten Staaten und Großbritannien, bedurft. Diese beiden Staaten übernahmen aber nur sehr beschränkt Verantwortung für die europäischen Sicherheits- und Wirtschaftsstrukturen. Locarno hatte gezeigt, was möglich war, wenn Großbritannien bereit war, sein Gewicht in die Waagschale zu werfen. Allerdings war Locarno auch einer der

\footnotetext{
${ }^{145}$ Siehe ibid. S. 94.

${ }^{146}$ Siehe Hoesch an AA (8.6.1926), PAAA R, 70494.

${ }^{147}$ Siehe RunderlaB Bülow (25.6.1927), ADAP B V, Nr. 255.

${ }^{148}$ Siehe SALZMANN, Großbritannien, S. 245; JACOBSON, Locarno Diplomacy, S. 388.
} 
wenigen Fälle gewesen, in denen London Verantwortung für die europäische Sicherheit (zumindest im Westteil Europas) übernommen hatte. Andere Sicherheitsinitiativen, multilateral wie bilateral, wurden gerade durch Großbritannien verhindert, wie das Beispiel des Genfer Protokolls zeigte. Auch die Vereinigten Staaten griffen nicht stabilisierend in die europäische Politik ein, es blieb bei einer einzigen ebenso großen wie leeren Geste, dem KelloggBriand-Pakt, der seine Entstehung jedoch gerade dem amerikanischen Wunsch verdankte, nicht in europäische Angelegenheiten involviert zu werden. Auf wirtschaftlichem Gebiet unternahmen die beiden angelsächsischen Mächte ebenfalls wenig, um die Lage zu verbessern. Die starre Haltung der USA in der Kriegsschuldenfrage (die direkte Auswirkungen auf das Reparationsproblem hatte) und die überaus problematische US-Handelspolitik verzögerten die wirtschaftliche Erholung Europas und beeinträchtigten dadurch auch die politische Annäherung ${ }^{149}$. Die Abstinenz Washingtons und Londons war um so tragischer, weil diese beiden Länder die Exponenten des liberalen Modells der Friedenssicherung waren. Es waren die Ideen Wilsons und der angelsächsischen Theoretiker des Wirtschaftsliberalismus wie Adam Smith und David Ricardo, die der modernen Politik der 1920er Jahre ihre Inspiration verliehen. Die Politik der beiden Länder blieb hinter diesen hohen Erwartungen zurück. Die Vereinigten Staaten und Großbritannien versagten als Führungskräfte des liberalen, modernen Systems der Friedenssicherung weitgehend.

Nicht nur die Haltung des Westens, sondern auch die Rolle der Sowjetunion behinderte die Modernisierung der Außenpolitik, und zwar in zweierlei Hinsicht. Sie störte das deutsch-französische Verhältnis erstens, weil sie Deutschland die wrussische Option « eröffnete, deshalb die deutsche Verständigungspolitik ständig relativierte und sie nur zu einer Option der deutschen Außenpolitik machte. Andererseits wurde der deutsche Handlungsspielraum durch die Rücksichtnahme auf die Beziehungen mit der Sowjetunion oft eingeschränkt ${ }^{150}$. Der Kommunismus und die aktive Unterstützung der kommunistischen Parteien in Europa durch Moskau trugen zweitens dazu bei, die innenpolitische Lage in Deutschland und Frankreich zu destabilisieren und erschwerten so die innenpolitische Mehrheitsfindung für eine moderne AuBenpolitik ${ }^{151}$.

$\mathrm{Zu}$ den Faktoren, die die Modernisierung der Außenpolitik behinderten, zählt natürlich auch die Weltwirtschaftskrise, die just zu dem Zeitpunkt zu

\footnotetext{
${ }^{149}$ Siehe JACOBSON, Locamo Diplomacy, S. $378 f$.

${ }^{150}$ Siehe ibid. S. 369.

${ }^{151}$ Für Frankreich siehe Pierre LEVEQUE, Histoire des forces politiques en France, Bd. 2: 1880-1940, Paris 1994, S. 202-207. Für Deutschland siehe Heinrich August WinKLER, Von der Revolution zur Stabilisierung. Arbeiter und Arbeiterbewegung in der Weimarer Republik 1918-1924, Bonn, Berlin 1984 (Geschichte der Arbeiter und der Arbeiterbewegung in Deutschland seit dem Ende des 18. Jahrhunderts, 9), S. $343 \mathrm{f}$.
} 
einem Aufflammen des wirtschaftlichen Nationalismus und der sozialen Spannungen führte, an dem sich das europäische Staatensystem und die deutsch-französischen Beziehungen in einer kritischen Phase befanden. Die Weltwirtschaftskrise mußte sich um so stärker auswirken, weil es in den 1920er Jahren, auch wegen des Reparations- und Kriegsschuldenproblems, kaum gelungen war, die wirtschaftlichen Probleme der Nachkriegszeit grundsätzlich zu lösen.

Die Modernisierung der Außenpolitik scheiterte also Ende der 1920er Jahren an einer Kumulierung hemmender Faktoren: In Deutschland und Frankreich blieb sie der Revisions- bzw. Sicherheitspolitik oft untergeordnet, die USA und Großbritannien wurden ihrer Führungsrolle nicht gerecht, der sowjetische Einfluß verschärfte innergesellschaftliche Spannungen, personelle Umwälzungen und die Weltwirtschaftskrise, die durch strukturelle ökonomische Probleme verstärkt wurde, trafen das internationale System zu einem Zeitpunkt, an dem der Modernisierungsproze $B$ selbst besonders verwundbar erschien, weil sich das internationale System in einer Phase der Neuorientierung und -bewertung von Zielen und Methoden befand.

$\mathrm{Da} B$ das liberale Friedensmodell aber durchaus das Potential für eine dauerhafte Befriedung hat, zeigt die Geschichte Westeuropas und der europäischen Integration nach 1945. Dies lag nicht zuletzt an den ungleich günstigeren Rahmenbedingungen nach dem Zweiten Weltkrieg: Nach der totalen Kriegsniederlage kam für Deutschland (zumindest für dessen westlichen Teil) nur eine Politik auf der Grundlage des liberalen Modells der Friedenssicherung in Frage, um überhaupt wieder auf die internationale Bühne zurückzukehren. Frankreich mußte aufgrund des amerikanischen und britischen Drucks und der Bedrohung durch die Sowjetunion von einer Politik Abstand nehmen, die auf die Zerschlagung Deutschlands ausgerichtet war. Vor allem die USA übernahmen nach dem Zweiten Weltkrieg die wirtschaftliche und politische Initiative, die sie nach 1918 hatten vermissen lassen. Der lange wirtschaftliche Aufschwung der zweiten Nachkriegszeit erleichterte die Aussöhnung zwischen den Völkern und ebnete den Weg für die Zusammenarbeit. Die Modernisierung der Außenpolitik, die in den Jahren 1923 bis 1929 ihren Anfang genommen hatte, fand so nach 1945 ihre Fortsetzung und Weiterentwicklung. 